\title{
Performance Evaluation of a Two-Phase Closed Thermosyphon Having a Converging-Diverging Pipe Body
}

\author{
Connor A.H. Bliss, Ali Tarokh* \\ Department of Mechanical Engineering, Lakehead University, Thunder Bay, Canada \\ *atarokh@lakeheadu.ca
}

\begin{abstract}
The present study focuses on improving the heat transfer performance of a two-phase closed thermosyphon (TPCT) by altering the geometry of a typical straight pipe thermosyphon body to have a converging-diverging section. The flow path of the liquid-vapor fluid mixture is augmented with this new design to induce thermal boundary layer mixing thereby enhancing the convection heat transfer within the system during operation. A multiphase numerical simulation model has been developed to simulate the fluid phase change and wall temperature distribution of a two-phase closed thermosyphon. The Lee model is used to calculate mass transfer source terms during the condensation and evaporation phase change processes and the Volume of Fluid (VOF) method is employed to track liquid-vapor interface movement during the simulations. Wall temperature distributions as well as overall thermal resistance values are compared with experimental values available in the literature in order to validate the simulation model for a straight pipe geometry. Two additional model geometries are then used for comparative study where the converging-diverging $(\mathrm{CD})$ section is positioned within the adiabatic section and condenser section respectively. The numerically simulated wall temperature distribution and overall thermal resistance results indicate that the most significant impact can be made when the CD section is positioned in the adiabatic section and condenser section exhibiting reduction of $1.7 \%$ and $3.4 \%$, respectively, in overall thermal resistance.
\end{abstract}

Keywords-Thermosyphon; Phase-change; Multiphase flow; Computational fluid dynamics; Numerical simulation;

\section{INTRODUCTION}

A two-phase closed thermosyphon (TPCT) is a form of wickless heat pipe capable of passive, efficient and rapid transport of thermal energy across its domain [1][2]. A wide variety of thermosyphon based designs are used in electronic equipment cooling [3], heat exchanger design [4] and renewable energy extraction [5]. Widely considered to be among the most efficient heat transfer systems available [6], a TPCT uses simultaneous evaporation and condensation phase change of the working fluid contained within the closed pipe body as a simple means for passively transporting heat from the hot end of the pipe to the cold end [7]. A typical TPCT has three main sections:

- Evaporator section, the lower portion of the body where heat is added through the walls to evaporate a pool of working fluid.

- Condenser section, the upper portion of the body with externally cooled walls to condense working fluid.

- Adiabatic section, thermally insulated portion of the body which separates the evaporator and condenser sections.

In the condenser and adiabatic sections, cooled liquid condensate flows along the inner walls of the pipe to the evaporator section due to gravity [8]. The thermal resistance of this condensing fluid, however, can lead to potential reduction in the heat transfer capacity and overall performance of a TPCT [9][10]. In order to mitigate TPCT performance issues, the focus of the present study is on numerically investigating the effectiveness of direct enhancement in the internal convective heat transfer within a TPCT.

Many research-works over the past decade have studied how TPCT performance can be affected by various physical operational parameters and conditions. Fadhl et al [11] investigated the effects which varied heat inputs and evaporator section filling ratios have on the overall thermal resistance of a water charged TPCT both experimentally and numerically. In their study, heat inputs ranged from $100.41 \mathrm{~W}$ to $376.14 \mathrm{~W}$ while filling ratios of $0.3,0.5$ and 0.8 were examined at each power input level. The optimal evaporator section filling ratio was determined to be $50 \%$ and the overall thermal resistance of the TPCT decreased at heat input levels above 170W. Aung et al. [12] used numerical models to explore the heat transfer performance of a closed loop TPCT operating at varied inclination angles and riser section diameters. In their study they concluded that the efficiency of their system coincides incrementally with heat input section orientation angle as well as the riser section diameter. Fertahi et al. [13] carried out a numerical study on the enhancement of thermal performance in a TPCT using tilted fins on the lateral surface of the condenser section as well as widening the diameter of the condenser 
section. The system performance was quantified by the thermosyphon efficiency, calculated as the ratio of heat transferred out of the condenser section to the heat input in the evaporator section. The efficiency of the model with a widened condenser section was $68.65 \%$, while the efficiency of the model with added fins was $84.71 \%$. Jouhara et al [14] completed an experimental study of a TPCT charged with two diphenyl based working fluids operating within a mediumtemperature range. Their results show that a small diameter TPCT can operate at temperatures above $400^{\circ} \mathrm{C}$ with relatively low thermal resistance. Mohamad et al. [15] developed a numerical model using the Lattice Boltzmann Method to simulate the flow of fluid across a von-Karman vortex generating obstacle in an heated open-ended channel. Results from their study show that vortex streets propagating in the wake of the obstacle induce a significant mixing effect amongst the laminar thermal boundary layers within the flowing fluid. Their study also concludes that one or two obstacles in a channel has minimal impact on the exhibited pressure drop across the domain.

It is clear that there has been meaningful development in this area of research, however, there still lacks clear investigation into the improvement of a TPCT by disturbing the flow regimes of the working fluid directly. Here, the focus is on improving the heat transfer performance of a TPCT by altering the geometry of a typical straight pipe thermosyphon to have a converging-diverging (CD) section. First, we examine the numerical treatment of the evaporation and condensation phase change processes, we then provide a thorough explanation of the mathematical models used for numerical simulation. Next, the operating parameters and solution methods are detailed, followed by the presentation of our simulation results.

\section{MethodOLOGY}

A two-dimensional simulation model has been developed to carry out a numerical study of the thermal transport performance of a TPCT with augmented geometry. In order to validate the simulation model, geometric parameters and operating conditions are based on the experimental work outlined in the study by Fadhl et al. [11]. Figure 1a shows the geometry of the straight wall thermosyphon body used for model validation and baseline simulation results. Figures $1 \mathrm{~b}$ and 1c show the geometry of the thermosyphon body with a converging-diverging wall section in the center of the adiabatic section and the lower portion of the condenser section, respectively. The dimensions of the converging-diverging section for both case 2 and case 3 were selected such that the throat is no less than $40 \%$ of the inner wall diameter, minimizing back-pressure caused by flow restriction through the CD section throat.

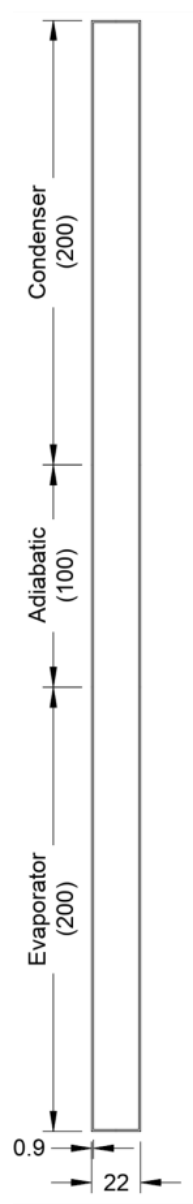

(a)

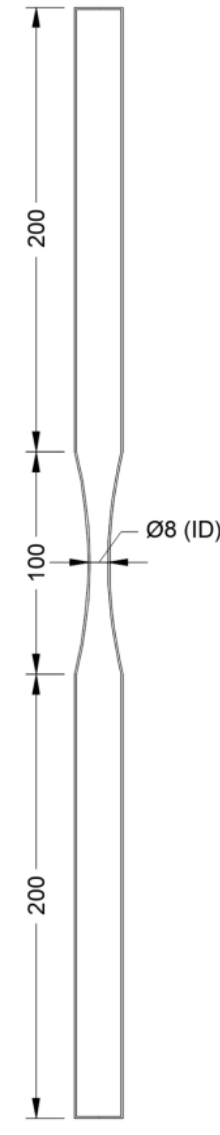

(b)

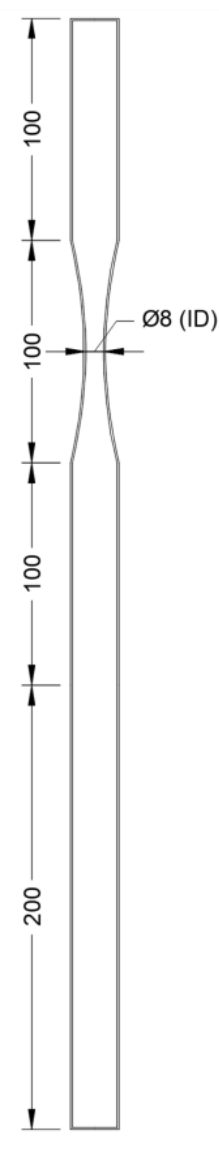

(c)
Figure 1. Thermosyphon geometry [all units in millimeters]. (a) Case 1: Baseline, (b) Case 2: CD in adiabatic section, (c) Case 3: CD in condenser section.

\section{A. Multiphase Volume of Fluid (VOF) Model}

Numerical simulation of the evaporation and condensation processes requires that the model accounts for the presence of a multiphase fluid. Using the volume of fluid (VOF) model, the volume fraction of each phase, the interface and it's curvature can be tracked on a Eulerian coordinate representation of the fluid domain [16]. In each cell, the sum of volume fractions for the vapour phase $\left(\alpha_{\mathrm{V}}\right)$ and liquid phase $\left(\alpha_{\mathrm{V}}\right)$ must maintain unity as follows:

$\alpha_{\mathrm{V}}+\alpha_{\mathrm{L}}=1$.

This implies that the continuity equation in the VOF model can be solved for the secondary phase only, while a single energy equation and a single momentum equation are solved at the mixture level using volume averaged parameters.

\section{Continuity Equation}

We begin by outlining the continuity equation solved in the VOF model:

$\frac{\partial}{\partial t}\left(\alpha_{L} \rho_{L}\right)+\nabla \cdot\left(\alpha_{L} \rho_{L} \vec{u}\right)=S_{M}$. 
Here, $\alpha_{\mathrm{L}}$ is the volume fraction of the liquid phase, $\rho_{L}$ is the density of the liquid phase, $\vec{u}$ is the velocity vector, and $S_{M}$ is the source term for mass as described by the corresponding phase change process in Section 2.2.

\section{Energy Equation}

The energy equation is then modelled as follows:

$\frac{\partial}{\partial t}(\rho e)+\nabla \cdot[\vec{u}(\rho e+\rho)]=\nabla \cdot(k \cdot \nabla T)+S_{E}$,

where $T$ is the mass-averaged temperature shared amongst phases, and $S_{E}$ is the source term for the energy equation, which is described by the corresponding phase change process in Section 2.2. $\rho$ is the density, and $k$ is the thermal conductivity. Both $\rho$ and $k$ are volume averaged parameters calculated as follows:

$\rho=\alpha_{V} \rho_{V}+\left(1-\alpha_{V}\right) \rho_{L}$

$k=\alpha_{V} k_{V}+\left(1-\alpha_{V}\right) k_{L}$.

The term $e$ in Equation (3), is the mass-averaged internal energy expressed as:

$e=\frac{\rho_{L} \alpha_{L} e_{L}+\rho_{V} \alpha_{V} e_{V}}{\rho_{L} \alpha_{L}+\rho_{V} \alpha_{V}}$

where $e_{L}$ and $e_{V}$ represent the specific internal energy of the liquid and vapour phases, respectively. These specific internal energy terms are defined by their respective caloric equation of state [17], which are calculated as:

$e_{L}=C_{p L}\left(T-T_{\text {sat }}\right)$

$e_{V}=C_{p V}\left(T-T_{\text {sat }}\right)$.

Here, $C_{p L}$ and $C_{p L}$ are the specific heat capacities of the liquid and vapour phases, respectively, and $T_{\text {sat }}$ is the saturation temperature of the fluid.

\section{Momentum Equation}

The conservation of momentum is modelled using:

$\frac{\partial}{\partial t}(\rho \vec{u})+\nabla\left(\rho \vec{u} \vec{u}^{T}\right)=-\nabla p+\nabla\left[\mu\left(\nabla \vec{u}+\nabla \vec{u}^{T}\right)\right]+\rho \vec{g}+\vec{F}_{C S F}$,

where $p$ is the local pressure, $\vec{g}$ is the acceleration due to gravity and $\mu$ is the volume-averaged viscosity,

$\mu=\alpha_{V} \mu_{V}+\left(1-\alpha_{V}\right) \mu_{L}$.

The $\vec{F}_{C S F}$ term in Equation (9) is the volumetric surface tension force calculated as a source term in the continuum surface force
(CSF) model proposed by Brackbill et al. [18]. It takes the form of:

$\vec{F}_{C S F}=2 \sigma_{l v}\left(\frac{\alpha_{L} \rho_{L} C_{V} \nabla \alpha_{V}+\alpha_{V} \rho_{V} C_{L} \nabla \alpha_{L}}{\rho_{V}+\rho_{L}}\right)$,

where $C_{V}$ and $C_{L}$ are the vapour surface and liquid surface curvatures defined in terms of the divergence of the unit normal [18], and $\sigma_{l v}$ is the surface tension coefficient.

\section{B. Phase Change Model}

To account for the mass and energy transfer during the evaporation-condensation phase change process, the following empirical expressions proposed by Lee [19] and De Schepper et al. [20] are used to calculate the source term for mass in Equation (2) and the source term for energy in Equation (3).

Mass source terms in the Evaporation process $\left(T_{\text {mix }}>T_{\text {sat }}\right)$ are calculated as follows:

Liquid Phase: $\quad S_{M}=-\beta_{e} \alpha_{l} \rho_{l}\left|\frac{T_{\text {mix }}-T_{\text {sat }}}{T_{\text {sat }}}\right|$

Vapour Phase: $\quad S_{M}=\beta_{e} \alpha_{l} \rho_{l}\left|\frac{T_{\text {mix }}-T_{\text {sat }}}{T_{\text {sat }}}\right|$

Mass source terms in the Condensation process $\left(T_{\text {mix }}<T_{\text {sat }}\right)$ are calculated as follows:

Liquid Phase: $\quad S_{M}=\beta_{c} \alpha_{v} \rho_{v}\left|\frac{T_{\text {mix }}-T_{\text {sat }}}{T_{\text {sat }}}\right|$

Vapour Phase: $\quad S_{M}=-\beta_{c} \alpha_{v} \rho_{v}\left|\frac{T_{\text {mix }}-T_{\text {sat }}}{T_{\text {sat }}}\right|$

Energy source term in the Evaporation process $\left(T_{\text {mix }}>T_{\text {sat }}\right)$ is calculated as follows:

Liquid Phase: $\quad S_{E}=-\beta_{e} \alpha_{l} \rho_{l}\left|\frac{T_{\text {mix }}-T_{s a t}}{T_{s a t}}\right| \cdot L H$

Energy source terms in the Condensation process $\left(T_{\text {mix }}<T_{\text {sat }}\right)$ is calculated as follows:

Vapour Phase: $S_{E}=\beta_{c} \alpha_{v} \rho_{v}\left|\frac{T_{m i x}-T_{s a t}}{T_{s a t}}\right| \cdot L H$.

In Equations (12-17), $T_{\text {mix }}$ is the mass averaged temperature shared amongst phases in a cell, and $T_{\text {sat }}$ is the prescribed saturation temperature for the working fluid in the system. $\mathrm{LH}$ in Equations (16) and (17) is the latent heat of the working fluid. $\beta_{e}$ and $\beta_{c}$ are the evaporation and condensation mass-transfer time relaxation factors, respectively calculated using Equations (18) and (19). Kim et al. [21] proposed that the calculation of the mass transfer time relaxation factors should be proportional to the ratio of phase densities for which a phase change is occurring: 
$\beta_{e}=0.1$

$\beta_{c}=\beta_{e}\left(\frac{\rho_{l}}{\rho_{v}}\right)$,

where $\beta_{e}$ and $\beta_{c}$ are the mass-transfer time relaxation parameters for the evaporation and condensation processes, $\rho_{L}$ is the density of the liquid phase, and $\rho_{v}$ is the density of the vapour phase.

\section{Grid Generation and Boundary Conditions}

The grid independence analysis was carried out using 4 different grid sizes based on the average wall temperature in the evaporator section. Variation in average evaporator section wall temperature is $0.01 \%$ from Grid 3 (123,210 quadrilateral elements) to Grid 4 (154,012 quadrilateral elements), which implies that Grid 3 is sufficient for capturing the wall temperature distributions. There is no appreciable difference in wall temperature resulting from larger grid sizing beyond that of Grid 3. As such, Grid 3 is used for the baseline (Case 1) and model validation study. The comparisons are shown in Table 1 , where $\mathrm{N}$ is the number of grid elements, and $\Delta T_{e, a v g}$ is the percent change in average evaporator section temperature between 2 consecutive grid size levels.

TABLE I. TABLE 1: GRID-INDEPENDENCE OF EVAPORATOR SECTION TEMPERATURE.

\begin{tabular}{|l|l|l|l|l|}
\hline \multicolumn{1}{|c|}{ Case } & \multicolumn{1}{c|}{ Grid 1 } & \multicolumn{1}{c|}{ Grid 2 } & \multicolumn{1}{c|}{ Grid 3 } & Grid 4 \\
\hline $\mathrm{N}$ & 61,605 & 92,408 & 123,210 & 154,012 \\
\hline $\boldsymbol{T}_{\boldsymbol{e}, \boldsymbol{a v g} \boldsymbol{g}}[\mathrm{K}]$ & 375.25 & 374.21 & 373.94 & 373.91 \\
\hline$\Delta \boldsymbol{T}_{\boldsymbol{e}, \boldsymbol{a v g} \boldsymbol{g}}[\mathrm{K}]$ & - & $0.27 \%$ & $0.07 \%$ & $0.01 \%$ \\
\hline
\end{tabular}

Figure 2 shows the grid cells and the spatial grid refinements. The inner wall requires a fine grid spacing to capture the condensation and evaporation phase change. Due to the nature of quadrilateral cell mapping, grid refinements were carried over into the corners of the domain. This did not pose any problems as the upper and lower walls are not used for energy exchange in the simulations.

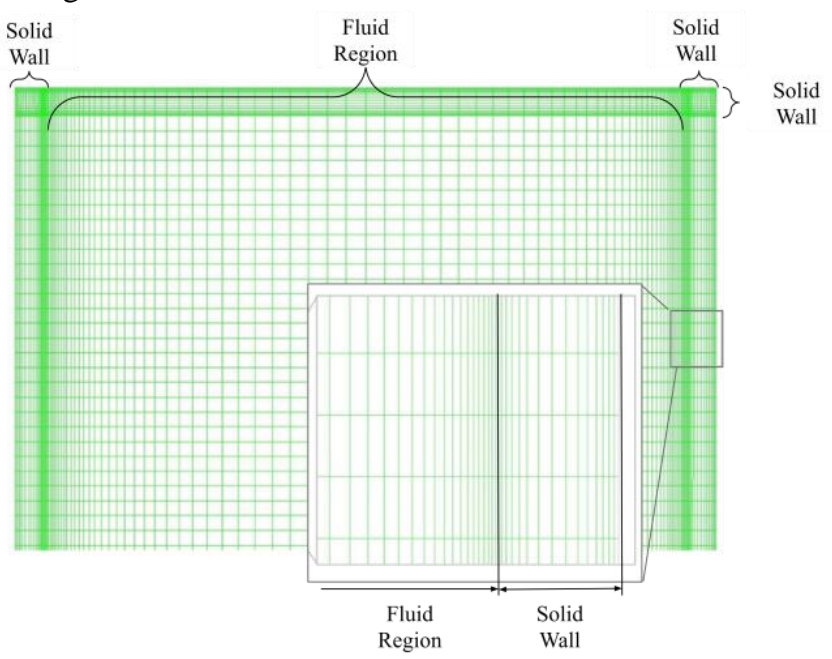

Figure 2. Computational grid, showing near wall refinement.
On all interior walls of the domain, a no-slip boundary is imposed. A constant heat flux condition of $940.35 \mathrm{~W} / \mathrm{m}^{2}$ is imposed on the vertical exterior walls of the evaporator section, which corresponds to a heat input of $376.14 \mathrm{~W}$. A zero-flux boundary condition is applied to the exterior walls of the adiabatic section and, the top and bottom exterior walls since these surfaces are considered to be insulated. A convection boundary condition is applied to the vertical exterior walls of the condenser section, where $1163.5 \mathrm{~W} / \mathrm{m}^{2} \mathrm{~K}$ is prescribed for the convection coefficient at the outer wall, and the cooling water jacket free stream temperature is $309.4 \mathrm{~K}$. Acceleration due to gravity is $-9.81 \mathrm{~m} / \mathrm{s}^{2}$ in the y-direction. The boundary and operating conditions correspond to values outlined in the experimental and CFD study of Fadhl et al. [11]. The liquid density and surface tension coefficient are based on the mass averaged temperature shared amongst each phase. As discussed in their study, Fadhl et al. [11] sourced these values using a steam-table as follows:

$\rho_{l}=859.0083+1.252209 T-0.0026429 T^{2}$

$\sigma_{l v}=0.098058-1.845 \times 10^{-5} T-2.3 \times 10^{-7} T^{2}$

where $\rho_{l}$ is the density of the liquid phase, $\sigma_{l v}$ is the surface tension coefficient. Temperature based properties in Equations (20-21) and all other constant material properties for each phase are specified in

TABLE II. MATERIAL PROPERTIES FOR VAPOUR AND LIQUID PHASE.

\begin{tabular}{|l|c|l|l|}
\hline \multirow{2}{*}{ Property } & \multirow{2}{*}[\text{Units}]{} & \multicolumn{2}{c|}{ Phase } \\
\cline { 3 - 4 } & & \multicolumn{1}{|c|}{ Water-Vapour } & Water-Liquid \\
\hline Order & - & Primary Phase & Secondary Phase \\
\hline Density & $\mathrm{kg} / \mathrm{m}^{3}$ & $\rho_{v}=0.5542$ & Equation $(20)$ \\
\hline Viscosity & $\mathrm{kg} /(\mathrm{ms})$ & $\mu_{v}=1.340 \times 10^{-5}$ & $\mu_{l}=1.003 \times 10^{-3}$ \\
\hline $\begin{array}{l}\text { Thermal } \\
\text { Conductivity }\end{array}$ & $\mathrm{kg} /(\mathrm{m} \mathrm{K})$ & $k_{v}=0.0261$ & $k_{l}=0.6$ \\
\hline Latent Heat & $\mathrm{J} / \mathrm{kg}$ & \multicolumn{2}{c|}{$\mathrm{LH}=2.455 \times 10^{6}$} \\
\hline Surface Tension & $\mathrm{N} / \mathrm{m}$ & \multicolumn{2}{|c|}{ Equation (21) } \\
\hline
\end{tabular}

\section{Operating Conditions}

An initial liquid volume fraction of 1.0 is patched to fluid cells in the lower $50 \%$ of the evaporator section, while the remaining cells are patched with a value of 0 . An initial temperature of $373.15 \mathrm{~K}$ is patched to the adiabatic and evaporator section walls and interior fluid region. The condenser walls are initialized with a temperature of $315 \mathrm{~K}$. An operating pressure of $101,325 \mathrm{~Pa}$ to simulate phase change at constant pressure in the Lee [19] model.

\section{E. Solution Methods and Convergence Strategy}

The time-dependent governing equations are integrated numerically using the finite volume method to approximate a transient solution. The variable time-stepping method is used with a maximum time step size of 0.0005 seconds to maintain a global courant number below 1.0. For transient start-up, a total of 30 seconds is simulated. The SIMPLE algorithm is specified for pressure-velocity coupling. Second-order upwind 
discretization is used for both the momentum and energy equations, while the implicit method is used for the volume fraction formulation in each of the simulation cases. The Compressive interface capturing scheme is chosen for volume fraction interpolation at the interface boundary. The convergence criteria for volume fraction and velocity residuals are $10^{-4}$, and $10^{-6}$ for energy residuals, at each time step.

\section{VALIDATION}

A validation study was carried out, using the baseline simulation (Case 1), by comparing wall temperature values for the condenser and evaporator sections, amongst experimental values reported by Fadhl et al. [11]. The average wall temperature in the evaporator section for the numerically simulated baseline (Case 1) was recorded as $T_{e, \text { avg-Num }}=$ $373.94 \mathrm{~K}$, whereas the experimental value was reported to be $T_{e, a v g-E x p}=370.20 \mathrm{~K}$. This corresponds to a change of $1.01 \%$ which is well below $10 \%$. In the condenser section, the average wall temperature for the numerically simulated baseline (Case 1) was $T_{c, a v g-N u m}=317.74 \mathrm{~K}$, and the experimental value was $T_{c, a v g-E x p}=330.32 \mathrm{~K}$, which corresponds to a difference of $3.81 \%$. While the variation between numerical and experimental evaporator section wall temperature is acceptable, there is a more considerable difference between the numerical and experimental results in the condenser section. This difference can be attributed to the timescale over which the numerical analysis was completed, at only half of the 60 seconds reported in the experimental study to reach steady operation. However, the difference below 5\% indicates that the numerical simulations provide sufficiently accurate results compared with the experimental data for the proof-of-concept analysis related to the design modifications considered here.

\section{RESULTS}

We begin by examining the average wall temperature of the condenser section, for each simulation case, shown in Figure 3. It can be seen that the wall temperature profiles approach pseudo-steady behavior after 10 seconds into the simulation period. Experimentally [11] and numerically obtained temperatures are presented in Table 3.

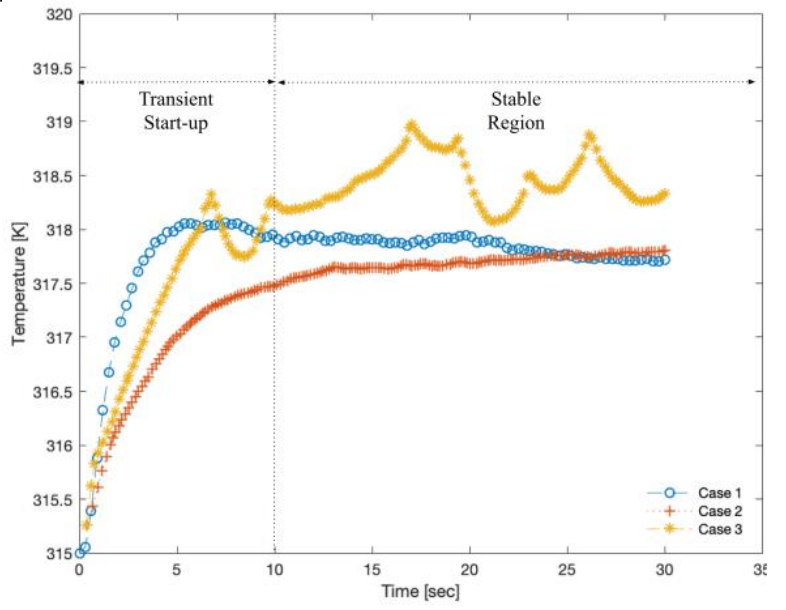

Figure 3. Evolution of condenser section wall temperatures during transient start-up and stable operation.
The start-up characteristics for each case do not vary significantly as each case rapidly approaches its respective operational temperature range. After start-up, Case 3 exhibited an oscillatory condenser section temperature profile, due to the $\mathrm{CD}$ section occupying the lower half of the condenser section forcing phase change to occur in this region. The numerically obtained temperature readings presented in Table 3 are timeaveraged temperatures, recorded after transient start-up (between 10s and 30s), as the TPCT approached a pseudosteady operational state. The overall effective thermal resistance of the TCPT is evaluated by comparing the average wall temperature values between the condenser and evaporator sections, then dividing that difference by the system heat input, expressed as:

$R_{e f f}=\frac{T_{e, a v g}-T_{c, a v g}}{\dot{Q}_{e}}$,

here, $\dot{Q}_{e}$ is the heat input in the evaporator section, $T_{e, \text { avg }}$ and $T_{c, a v g}$ are averaged wall temperatures for the evaporator and condenser sections, respectively.

TABLE III. AVERAGED SECTION WALL TEMPERATURES AT $30 \mathrm{~s}$.

\begin{tabular}{|c|l|l|l|l|}
\hline \multicolumn{2}{|c|}{} & Case 1 & Case 2 & Case 3 \\
\hline Evaporator & $T_{e, a v g}[K]$ & 373.94 & 372.83 & 372.62 \\
\hline Adiabatic & $T_{a, a v g}[K]$ & 362.63 & 356.28 & 353.11 \\
\hline Condenser & $T_{c, a v g}[K]$ & 317.74 & 317.69 & 318.46 \\
\hline $\begin{array}{c}\text { Overall Effective } \\
\text { Thermal Resistance }\end{array}$ & $R_{\text {eff }}[K / W]$ & 0.1494 & 0.1466 & 0.1440 \\
\hline
\end{tabular}

The overall thermal resistance of the simulated thermosyphon cases with a converging-diverging section (Cases 2-3) are compared with the baseline simulation (Case 1, straight pipe body) by percent change comparison presented in Figure 4.

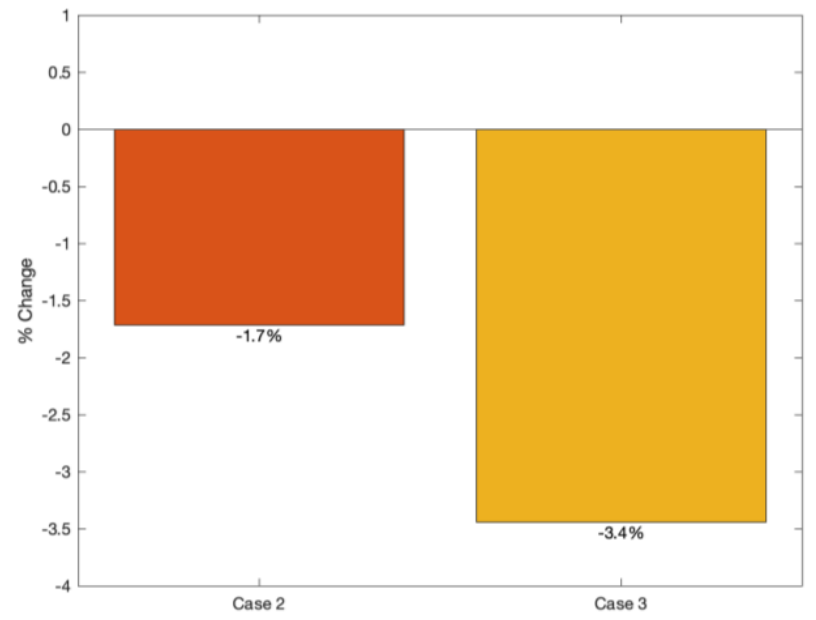

Figure 4. Percent change of overall thermal resistance for each case as compared to baseline (Case 1).

The most substantial reduction of overall thermal resistance, as compared with the baseline simulation (Case 1), was $-3.4 \%$ for the simulation model with a $\mathrm{CD}$ section positioned in the condenser lower half of the condenser section (Case 3). Next, the simulation model with a $\mathrm{CD}$ section positioned in the 
adiabatic section (Case 2) exhibited a $1.7 \%$ reduction in overall thermal resistance.

\section{CONCLUSIONS}

The performance enhancement of a two-phase closed thermosyphon with a converging-diverging wall section is investigated. The underlying theory is that by simply augmenting the flow path of the working fluid within a TPCT, the overall thermal resistance of such a system can be reduced by mixing thermally resistive boundary layers. Numerical simulations were employed to model heat transfer during the phase change processes of condensation and evaporation using the volume of fluid method and the Lee [19] model. Three simulations were compared to find the optimal positioning of the $\mathrm{CD}$ section within the system, quantified by the effects on wall temperature distribution and thermal resistance during transient thermosyphon operation. A baseline simulation of a straight pipe geometry was developed to validate the simulation parameters for phase change heat transfer during evaporation and condensation compared to experiments. The phase change model produced a wall temperature distribution that agreed with experimental data [11] with a difference of $1.01 \%$ in the evaporator section and $3.81 \%$ in the condenser section. The validated simulation model was then used for two additional modified geometries. A converging-diverging section was positioned in the center of the adiabatic section, which resulted in a $1.7 \%$ reduction in overall thermal resistance. Next, the converging-diverging section was positioned in the lower half of the condenser section, which led to a $3.4 \%$ reduction in overall thermal resistance. Through analysis, the effects which the converging-diverging section had on the fluid flow regimes in the domain agreed with the underlying theory. Acceleration of fluid through the throat of the $\mathrm{CD}$ section introduces turbulence to the flow thereby mixing thermal boundary layers. The present study has shown that potential degradation of TPCT performance can be helped by introducing a simple, unique, and effective design change with means of further improving the heat transfer capabilities of such devices.

\section{REFERENCES}

[1] A. Faghri, "Heat Pipes: Review, Opportunities and Challenges," Front. Heat Pipes, vol. 5, no. 1, 2014.

[2] T. P. Cotter, "Theroy of Heat Pipes," Los Alamos, Report No: LA3246-MS, 1965.

[3] M. H. A. Elnaggar and E. Edwan, "Heat Pipes for Computer Cooling Applications," Electron. Cool., 2016.

[4] L. Ling, Q. Zhang, Y. Yu, and S. Liao, "Experimental investigation on the thermal performance of water cooled multi-split heat pipe system (MSHPS) for space cooling in modular data centers," Appl. Therm. Eng., vol. 107, no. 2016, pp. 591-601, 2016.
[5] I. Carvajal-Mariscal, F. Sanchez-Silva, and G. Polup, "Development of High Efficiency Two-Phase Thermosyphons for Heat Recovery," Heat Exch. - Basics Des. Appl., no. March, 2012.

[6] M. Esen and H. Esen, "Experimental investigation of a two-phase closed thermosyphon solar water heater," Sol. Energy, vol. 79, no. 5, pp. 459-468, 2005.

[7] R. Parand, B. Rashidian, A. Ataei, and K. Shakiby, "Modeling the transient response of the thermosyphon heat pipes," J. Appl. Sci., vol. 9, no. 8, pp. 1531-1537, 2009.

[8] J. Legierski, B. Więcek, and G. De Mey, "Measurements and simulations of transient characteristics of heat pipes," Microelectron. Reliab., vol. 46, no. 1, pp. 109-115, 2006.

[9] M. Abu-Orabi, "Modeling of heat transfer in dropwise condensation," Int. J. Heat Mass Transf., vol. 41, no. 1, pp. 81-87, 1998.

[10] J. Xu, "Theoretical And Experimental Analysis Of Two-Phase Closed Thermosyphons," University of Alaska Fairbanks, 2008.

[11] B. Fadhl, L. C. Wrobel, and H. Jouhara, "Numerical modelling of the temperature distribution in a two-phase closed thermosyphon," Appl. Therm. Eng., vol. 60, no. 1-2, pp. 122-131, 2013.

[12] N. Z. Aung and S. Li, "Numerical investigation on effect of riser diameter and inclination on system parameters in a two-phase closed loop thermosyphon solar water heater," Energy Convers. Manag., vol. 75, pp. 25-35, 2013.

[13] S. E. D. Fertahi, T. Bouhal, Y. Agrouaz, T. Kousksou, T. El Rhafiki, and Y. Zeraouli, "Performance optimization of a two-phase closed thermosyphon through CFD numerical simulations," Appl. Therm. Eng., 2018.

[14] H. Jouhara and A. J. Robinson, "An Experimental Study of SmallDiameter Wickless Heat Pipes Operating in the Temperature Range $200^{\circ} \mathrm{C}$ to $450^{\circ} \mathrm{C}$," Heat Transf. Eng., vol. 30 , no. 13, pp. 1041-1048, Nov. 2009.

[15] A. A. Mohamad, A. Tarokh, and H. Alansary, "Heat transfer enhancement of laminar forced convection in a channel by Von-Karman vortex generator," Prog. Comput. Fluid Dyn., vol. 16, no. 5, pp. 334-338, 2016

[16] C. W. Hirt and B. D. Nichols, "Volume of fluid (VOF) method for the dynamics of free boundaries," J. Comput. Phys. Comput. Phys., vol. 39, no. 1, pp. 201-225, 1981.

[17] H. Jouhara and A. J. Robinson, "Experimental investigation of small diameter two-phase closed thermosyphons charged with water, FC-84, FC-77 and FC-3283," Appl. Therm. Eng., vol. 30, no. 2-3, pp. 201-211, 2010.

[18] J. U. Brackbill, D. B. Kothe, and C. Zemach, "A continuum method for modeling surface tension,” J. Comput. Phys., vol. 100, no. 2, pp. 335354, 1992.

[19] W. H. Lee, "PRESSURE ITERATION SCHEME FOR TWOPHASE FLOW MODELING.," Multi-phase Transp. Fundam. React. Safety, Appl., vol. 1, pp. 407-431, 1980.

[20] S. C. K. De Schepper, G. J. Heynderickx, and G. B. Marin, "Modeling the evaporation of a hydrocarbon feedstock in the convection section of a steam cracker," Comput. Chem. Eng., vol. 33, no. 1, pp. 122 132, 2009.

[21] Y. Kim, J. Choi, S. Kim, and Y. Zhang, "Effects of mass transfer time relaxation parameters on condensation in a thermosyphon," J. Mech. Sci. Technol., 2015. 